\title{
Genetic impact of methylenetetrahydrofolate reductase (MTHFR) polymorphism on the susceptibility to colorectal polyps: a meta- analysis
}

\author{
Manyi Sun ${ }^{1 *}$ (D) Jin Zhong ${ }^{2}$, Li Zhang ${ }^{3}$ and Songli Shi ${ }^{3}$
}

\begin{abstract}
Background: There are several studies with inconsistent conclusions regarding the association between the rs1801133 and rs1801131 polymorphisms within the MTHFR (methylenetetrahydrofolate reductase) gene and colorectal polyp risk. This discrepancy led us to assess the genetic impact of the two polymorphisms on the susceptibility to colorectal polyps.
\end{abstract}

Methods: A meta-analysis was carried out for quantitative synthesis. According to the inclusion/exclusion criteria, we retrieved, screened and selected all published articles related to colorectal polyps and the MTHFR rs1801133 and rs1801131 polymorphisms. The $P$ value of association test, RRs (risk ratios) and 95\% Cls (confidence intervals) were mainly produced.

Results: A total of twenty-three case-control studies were included from twenty-two eligible articles. Pooling the results of both rs1801133 and rs 1801131 polymorphisms in the overall population suggested a nonsignificant association between colorectal polyp cases and controls, in that all $P$ values in the test of association were larger than 0.05. Nevertheless, pooling results in the "UK" subgroup of rs 1801131, comprising five studies (1257 cases/1407 controls), indicated an elevated risk in colorectal polyp cases in comparison with controls, under the genetic models of $C C$ vs. $\mathrm{AA}(P=0.032, \mathrm{RR}=1.27,95 \% \mathrm{Cls}=1.02,1.57)$ and $\mathrm{CC}$ vs. $\mathrm{AA}+\mathrm{AC}(P=0.036, \mathrm{RR}=1.27,95 \% \mathrm{Cls}=1$. 02, 1.60).

Conclusion: The C/C genotype of MTHFR rs1801131 is more likely to be a genetic risk factor for colorectal polyps in the UK region, although this finding should be verified with a larger sample size.

Keywords: MTHFR, Polymorphism, Colorectal polyps, Susceptibility

\section{Background}

Colorectal polyps exhibit different morphologic features with flat, depressed, serrated, sessile or pedunculated shapes and are often regarded as benign protrusions of the colon and rectum mucosa $[1,2]$. There are many types of colorectal polyps, such as hyperplastic polyps and adenomatous polyps $[2,3]$. Despite the low malignant potential, the

\footnotetext{
* Correspondence: qwe_11344@163.com

'Department of Gastroenterology, Tianjin Union Medical Center, No.190, Jieyuan Road, Hongqiao District, Tianjin 300121, People's Republic of China Full list of author information is available at the end of the article
}

possible malignant change in colorectal polyps is related to the presence of colorectal cancer (CRC). For instance, some colonic polyps exist in patients with familial adenomatous polyposis (FAP) who are prone to cancer [4].

The 5,10-methylenetertahydrofolate reductase (MTHFR) gene is essential for the folate cycle and homocysteine metabolism [5]. rs1801133 (C677T) and rs1801131 (A1298C) are two common functional polymorphisms within the MTHFR gene [6, 7]. MTHFR rs1801133 and rs1801131 polymorphisms were reportedly associated with an enhanced risk of colorectal adenomatous polyp patients in

(c) The Author(s). 2019 Open Access This article is distributed under the terms of the Creative Commons Attribution 4.0 International License (http://creativecommons.org/licenses/by/4.0/), which permits unrestricted use, distribution, and reproduction in any medium, provided you give appropriate credit to the original author(s) and the source, provide a link to the Creative Commons license, and indicate if changes were made. The Creative Commons Public Domain Dedication waiver (http://creativecommons.org/publicdomain/zero/1.0/) applies to the data made available in this article, unless otherwise stated. 
the Korean population [8]. However, no association between the MTHFR rs1801133 polymorphism and colorectal adenomatous polyp susceptibility was reported in the Dutch [9] or Japanese population [10]. These findings merit a comprehensive evaluation.

To the best of our knowledge, only one reported meta-analysis [6] of the association between MTHFR rs1801131 and colorectal adenoma and three meta-analyses [6, 11, 12] of MTHFR rs1801133 and colorectal adenoma were found during the database searching. However, the conclusion remains inconsistent. Additionally, we failed to retrieve a meta-analysis specific for the association between MTHFR polymorphisms and the susceptibility to both hyperplastic/adenomatous polyps. Herein, we have made an attempt to better investigate the potential genetic role of MTHFR rs1801133 and rs1801131 polymorphisms in the risk of colorectal polyps through an updated meta-analysis.

\section{Methods}

\section{Database searching and screening process}

Two authors (MS and JZ) gathered the relative records through searching the databases, namely, PubMed, WOS (Web of Science), and EMBASE (Excerpta Medica Database), prior to March 2018. The PRISMA (Preferred Reporting Items for Systematic Reviews and Meta-Analyses) guidelines were followed [13]. The search terms used with the databases are shown in Additional file 1: Table S1. We independently excluded duplicate and ineligible records based on the following criteria: reviews, mouse data, case reports or trials, meta-analyses, meeting or conference abstracts, other genes, non-SNP or nonpolyp data, or missing genotype data for rs1801133 or rs1801131. Then, the remaining studies were included as eligible case-control studies.

\section{Data extraction and quality assessment}

We carefully extracted the data from the above selected studies. The chi-squared test was applied for the calculation of the $P$ value of HWE (Hardy-Weinberg Equilibrium). The included studies should provide the genotype frequency data of the control group, which also must be in line with the requirement of HWE. We summarized the main features of the included studies, such as first author name, publication year, polymorphism genotype frequency, country, ethnicity, genotyping assay, and $P$ value of HWE. We also utilized quality assessment (Newcastle-Ottawa Scale, NOS) to determine the quality score of the enrolled studies. Studies with poor quality (NOS score less than five) were excluded.

\section{Association test}

We obtained the $P_{\text {association, }}$, risk ratios (RRs) and 95\% confidence intervals (CIs) through the association test.
The $P_{\text {heterogeneity }}$ value of Cochran's Q statistic $>0.1$ or $\mathrm{I}^{2}$ value $<50 \%$ led us to use a fixed-effects model. Six genetic models were used: allele T vs. allele C for rs1801133, allele C vs. allele A for rs1801131 (allele); TT vs. CC, CC vs. AA (homozygote); CT vs. CC, AC vs. AA (heterozygote); $\mathrm{CT}+\mathrm{TT}$ vs. CC, $\mathrm{AC}+\mathrm{CC}$ vs. AA (dominant); TT vs. $\mathrm{CC}+\mathrm{CT}, \mathrm{CC}$ vs. $\mathrm{AA}+\mathrm{AC}$ (recessive); carrier $\mathrm{T}$ vs. carrier $\mathrm{C}$, carrier $\mathrm{C}$ vs. carrier $\mathrm{A}$ (carrier).

\section{Heterogeneity source analysis}

We also carried out a sensitivity analysis and subgroup analyses for all genetic models to evaluate the data stability and source of heterogeneity. Briefly, we omitted each included study in turn to acquire a group of meta-analysis estimations. The omitted study was regarded as the probable heterogeneity source if we detected an obvious alteration of RR and 95\% CI value. Subgroup analyses were also carried out, taking the factors of country, ethnicity (Caucasian/Asian) and disease type (hyperplastic polyps/ adenomatous polyps) into consideration.

\section{Publication bias analysis}

We conducted both Begg's test (Begg's funnel plot) and Egger's test (Egger's publication bias plot) to evaluate possible publication bias. The absence of a large publication bias was considered when the $P$ values of Begg's test and Egger's test were $>0.05$. STATA/SE software (StataCorp, USA) was utilized for all the above tests.

\section{Results}

\section{Identification of eligible studies}

We initially identified a total of 153 records by searching three databases, namely, PubMed $(n=22)$, WOS $(n=$ $83)$, and EMBASE $(n=48)$. After excluding duplicate records, a total of 115 records were filtered by our criteria. The following 88 records were excluded: reviews $(n=$ $31)$, mouse data $(n=4)$, case reports or trials $(n=7)$, meta-analyses $(n=6)$, meeting or conference abstracts $(\mathrm{n}=8)$; other genes $(n=9)$, non-SNP or nonpolyp data $(n=23)$. Subsequently, twenty-seven full-text articles were evaluated for eligibility. Five articles lacked control or T/T genotype data. Finally, a total of twenty-two articles $[8-10,14-32]$ were selected. We listed the characteristics of eligible studies in the meta-analysis (Table 1). The genotype contributions of all controls in the studies fulfilled the principle of HWE. We found that one article contained two case-control studies, namely, the genotype distribution data in both adenomatous and hyperplastic polyps. In total, twenty-three case-control studies were ultimately included for the overall meta-analysis of MTHFR rs1801133, and ten case-control studies were included for that of MTHFR rs1801131. In addition, one 
Table 1 Main features of eligible studies for pooled analysis

\begin{tabular}{|c|c|c|c|c|c|c|c|c|c|c|c|c|c|c|}
\hline \multirow[t]{2}{*}{ First author } & \multirow[t]{2}{*}{ Year } & \multirow[t]{2}{*}{ NOS } & \multirow[t]{2}{*}{ Polymorphism } & \multicolumn{3}{|c|}{ Case } & \multirow[t]{2}{*}{ Disease type } & \multicolumn{3}{|c|}{ Control } & \multirow[t]{2}{*}{ Country } & \multirow[t]{2}{*}{ Ethnicity } & \multirow[t]{2}{*}{ Genotyping assay } & \multirow[t]{2}{*}{$P_{H W E}$} \\
\hline & & & & $\begin{array}{l}\bar{A} / \\
A\end{array}$ & $A / B$ & $B / B$ & & $\mathrm{~A} / \mathrm{A}$ & $A / B$ & $B / B$ & & & & \\
\hline \multirow{4}{*}{$\begin{array}{l}\text { Al- } \\
\text { Ghnaniem } \\
{[14]}\end{array}$} & \multirow[t]{4}{*}{2007} & \multirow[t]{4}{*}{7} & rs1801133 & 22 & 12 & 1 & $\begin{array}{l}\text { adenomatous } \\
\text { polyps }\end{array}$ & 41 & 29 & 6 & UK & Caucasian & PCR-RFLP & 0.784 \\
\hline & & & rs1801133 & 11 & 3 & 3 & $\begin{array}{l}\text { hyperplastic } \\
\text { polyps }\end{array}$ & 41 & 29 & 6 & UK & Caucasian & PCR-RFLP & 0.784 \\
\hline & & & rs1801131 & 18 & 12 & 5 & $\begin{array}{l}\text { adenomatous } \\
\text { polyps }\end{array}$ & 47 & 26 & 3 & UK & Caucasian & PCR-RFLP & 0.799 \\
\hline & & & rs1801131 & 8 & 7 & 2 & $\begin{array}{l}\text { hyperplastic } \\
\text { polyps }\end{array}$ & 47 & 26 & 3 & UK & Caucasian & PCR-RFLP & 0.799 \\
\hline $\begin{array}{l}\text { Ashktorab } \\
{[15]}\end{array}$ & 2007 & 6 & rs1801133 & 18 & 4 & 0 & $\begin{array}{l}\text { colorectal } \\
\text { polyps }\end{array}$ & 30 & 5 & 0 & USA & Caucasian & PCR-RFLP & 0.649 \\
\hline \multirow[t]{2}{*}{ Beckett [16] } & \multirow[t]{2}{*}{2015} & \multirow[t]{2}{*}{5} & rs1801133 & 29 & 20 & 7 & $\begin{array}{l}\text { adenomatous } \\
\text { polyps }\end{array}$ & 88 & 91 & 18 & Australia & Caucasian & PCR-RFLP & 0.421 \\
\hline & & & rs1801131 & 28 & 22 & 6 & $\begin{array}{l}\text { adenomatous } \\
\text { polyps }\end{array}$ & 101 & 83 & 13 & Australia & Caucasian & PCR-RFLP & 0.460 \\
\hline Chen [17] & 1998 & 8 & rs1801133 & 102 & 126 & 30 & $\begin{array}{l}\text { adenomatous } \\
\text { polyps }\end{array}$ & 323 & 324 & 66 & USA & Caucasian & PCR-RFLP & 0.234 \\
\hline Chiang [18] & 2015 & 7 & rs1801133 & 44 & 26 & 0 & $\begin{array}{l}\text { adenomatous } \\
\text { polyps }\end{array}$ & 91 & 73 & 18 & China & Asian & PCR-RFLP & 0.553 \\
\hline $\begin{array}{l}\text { de Vogel } \\
{[19]}\end{array}$ & 2011 & 6 & rs1801133 & 947 & 714 & 135 & $\begin{array}{l}\text { adenomatous } \\
\text { polyps }\end{array}$ & 4463 & 3563 & 708 & Norway & Caucasian & Real-time PCR & 0.933 \\
\hline Delgado [20] & 2001 & 8 & rs1801133 & 6 & 19 & 7 & $\begin{array}{l}\text { adenomatous } \\
\text { polyps }\end{array}$ & 34 & 52 & 24 & Mexico & Caucasian & PCR-RFLP & 0.625 \\
\hline \multirow[t]{2}{*}{$\begin{array}{l}\text { Giovannucci } \\
\text { [21] }\end{array}$} & \multirow[t]{2}{*}{2003} & \multirow[t]{2}{*}{6} & rs1801133 & 157 & 168 & 49 & $\begin{array}{l}\text { adenomatous } \\
\text { polyps }\end{array}$ & 299 & 325 & 101 & USA & Caucasian & PCR-RFLP & 0.401 \\
\hline & & & rs1801131 & 186 & 165 & 24 & $\begin{array}{l}\text { adenomatous } \\
\text { polyps }\end{array}$ & 369 & 299 & 57 & USA & Caucasian & PCR-RFLP & 0.740 \\
\hline Goode [22] & 2004 & 7 & rs1801133 & 236 & 196 & 58 & $\begin{array}{l}\text { adenomatous } \\
\text { polyps }\end{array}$ & 259 & 238 & 67 & USA & Caucasian & PCR-RFLP & 0.281 \\
\hline \multirow[t]{2}{*}{ Hazra [23] } & \multirow[t]{2}{*}{2007} & \multirow[t]{2}{*}{7} & rs1801133 & 217 & 245 & 63 & $\begin{array}{l}\text { adenomatous } \\
\text { polyps }\end{array}$ & 229 & 232 & 64 & USA & Caucasian & NA & 0.658 \\
\hline & & & rs1801131 & 278 & 211 & 48 & $\begin{array}{l}\text { adenomatous } \\
\text { polyps }\end{array}$ & 264 & 219 & 46 & USA & Caucasian & NA & 0.951 \\
\hline Hirose [24] & 2005 & 8 & rs1801133 & 182 & 203 & 67 & $\begin{array}{l}\text { adenomatous } \\
\text { polyps }\end{array}$ & 399 & 496 & 155 & Japan & Asian & PCR-RFLP & 0.966 \\
\hline \multirow[t]{2}{*}{ Yi [8] } & \multirow[t]{2}{*}{2006} & \multirow[t]{2}{*}{6} & rs1801133 & 5 & 5 & 4 & $\begin{array}{l}\text { adenomatous } \\
\text { polyps }\end{array}$ & 2 & 4 & 0 & Korea & Asian & PCR-RFLP & 0.221 \\
\hline & & & rs1801131 & 10 & 3 & 1 & $\begin{array}{l}\text { adenomatous } \\
\text { polyps }\end{array}$ & 3 & 3 & 0 & Korea & Asian & PCR-RFLP & 0.414 \\
\hline Levine [25] & 2000 & 7 & rs1801133 & 256 & 163 & 52 & $\begin{array}{l}\text { adenomatous } \\
\text { polyps }\end{array}$ & 263 & 198 & 49 & USA & Caucasian & PCR-RFLP & 0.193 \\
\hline \multirow[t]{2}{*}{$\begin{array}{l}\text { Lightfoot } \\
{[26]}\end{array}$} & \multirow[t]{2}{*}{2008} & 8 & rs1801133 & 135 & 132 & 41 & $\begin{array}{l}\text { adenomatous } \\
\text { polyps }\end{array}$ & 130 & 139 & 27 & UK & Caucasian & $\begin{array}{l}\text { Taqman drug } \\
\text { metabolizing } \\
\text { genotyping assays }\end{array}$ & 0.238 \\
\hline & & & rs1801131 & 155 & 124 & 29 & $\begin{array}{l}\text { adenomatous } \\
\text { polyps }\end{array}$ & 140 & 130 & 26 & UK & Caucasian & $\begin{array}{l}\text { Taqman drug } \\
\text { metabolizing } \\
\text { genotyping assays }\end{array}$ & 0.590 \\
\hline $\begin{array}{l}\text { Marugame } \\
{[10]}\end{array}$ & 2000 & 8 & rs1801133 & 83 & 92 & 30 & $\begin{array}{l}\text { adenomatous } \\
\text { polyps }\end{array}$ & 89 & 105 & 26 & Japan & Asian & PCR-RFLP & 0.555 \\
\hline Mitrou [27] & 2006 & 7 & rs1801133 & 405 & 376 & 87 & $\begin{array}{l}\text { adenomatous } \\
\text { polyps }\end{array}$ & 402 & 407 & 89 & UK & Caucasian & PCR-RFLP & 0.340 \\
\hline & & & rs1801131 & 383 & 375 & 104 & $\begin{array}{l}\text { adenomatous } \\
\text { polyps }\end{array}$ & 415 & 380 & 88 & UK & Caucasian & PCR-RFLP & 0.941 \\
\hline
\end{tabular}


Table 1 Main features of eligible studies for pooled analysis (Continued)

\begin{tabular}{|c|c|c|c|c|c|c|c|c|c|c|c|c|c|c|}
\hline \multirow[t]{2}{*}{ First author } & \multirow[t]{2}{*}{ Year } & \multirow[t]{2}{*}{ NOS } & \multirow[t]{2}{*}{ Polymorphism } & \multicolumn{3}{|c|}{ Case } & \multirow[t]{2}{*}{ Disease type } & \multicolumn{3}{|c|}{ Control } & \multirow{2}{*}{ Country } & \multirow[t]{2}{*}{ Ethnicity } & \multirow[t]{2}{*}{ Genotyping assay } & \multirow[t]{2}{*}{$P_{H W E}$} \\
\hline & & & & $\begin{array}{l}\mathrm{A} / \\
\mathrm{A}\end{array}$ & $A / B$ & $\mathrm{~B} / \mathrm{B}$ & & $\mathrm{A} / \mathrm{A}$ & $A / B$ & $B / B$ & & & & \\
\hline \multirow[t]{2}{*}{ Pufulete [28] } & \multirow[t]{2}{*}{2003} & \multirow[t]{2}{*}{7} & rs1801133 & 20 & 13 & 2 & $\begin{array}{l}\text { adenomatous } \\
\text { polyps }\end{array}$ & 41 & 29 & 6 & UK & Caucasian & PCR-RFLP & 0.784 \\
\hline & & & rs1801131 & 18 & 12 & 5 & $\begin{array}{l}\text { adenomatous } \\
\text { polyps }\end{array}$ & 47 & 26 & 3 & UK & Caucasian & PCR-RFLP & 0.799 \\
\hline Ulrich [29] & 1999 & 9 & rs1801133 & 258 & 219 & 50 & $\begin{array}{l}\text { adenomatous } \\
\text { polyps }\end{array}$ & 303 & 269 & 73 & USA & Caucasian & PCR-RFLP & 0.260 \\
\hline Ulrich [30] & 2000 & 7 & rs1801133 & 98 & 72 & 26 & $\begin{array}{l}\text { hyperplastic } \\
\text { polyps }\end{array}$ & 297 & 258 & 71 & USA & Caucasian & PCR-RFLP & 0.192 \\
\hline van den [9] & 2005 & 7 & rs1801133 & 343 & 346 & 79 & $\begin{array}{l}\text { adenomatous } \\
\text { polyps }\end{array}$ & 325 & 305 & 79 & Netherlands & Caucasian & PCR-RFLP & 0.560 \\
\hline Williams [31] & 2013 & 7 & rs1801133 & 34 & 48 & 8 & $\begin{array}{l}\text { adenomatous } \\
\text { polyps }\end{array}$ & 44 & 42 & 9 & UK & Caucasian & PCR-RFLP & 0.822 \\
\hline \multirow[t]{2}{*}{ Yamaji [32] } & \multirow[t]{2}{*}{2009} & \multirow[t]{2}{*}{6} & rs1801133 & 263 & 325 & 124 & $\begin{array}{l}\text { adenomatous } \\
\text { polyps }\end{array}$ & 219 & 324 & 120 & Japan & Asian & TaqMan PCR & 0.993 \\
\hline & & & rs1801131 & 452 & 228 & 32 & $\begin{array}{l}\text { adenomatous } \\
\text { polyps }\end{array}$ & 441 & 197 & 25 & Japan & Asian & TaqMan PCR & 0.609 \\
\hline
\end{tabular}

A/A C/C genotype of rs1801133, or A/A genotype of $\mathrm{rs} 1801131, A / B C / T$ genotype of $r s 1801133$, or $A / C$ genotype of rs $1801131, B / B$ T/T genotype of $r s 1801133$, or C/C genotype of rs1801131, NA not available, PCR-RFLP polymerase chain reaction-restriction fragment length polymorphism, HWE Hardy-Weinberg Equilibrium, NOS Newcastle-Ottawa Scale
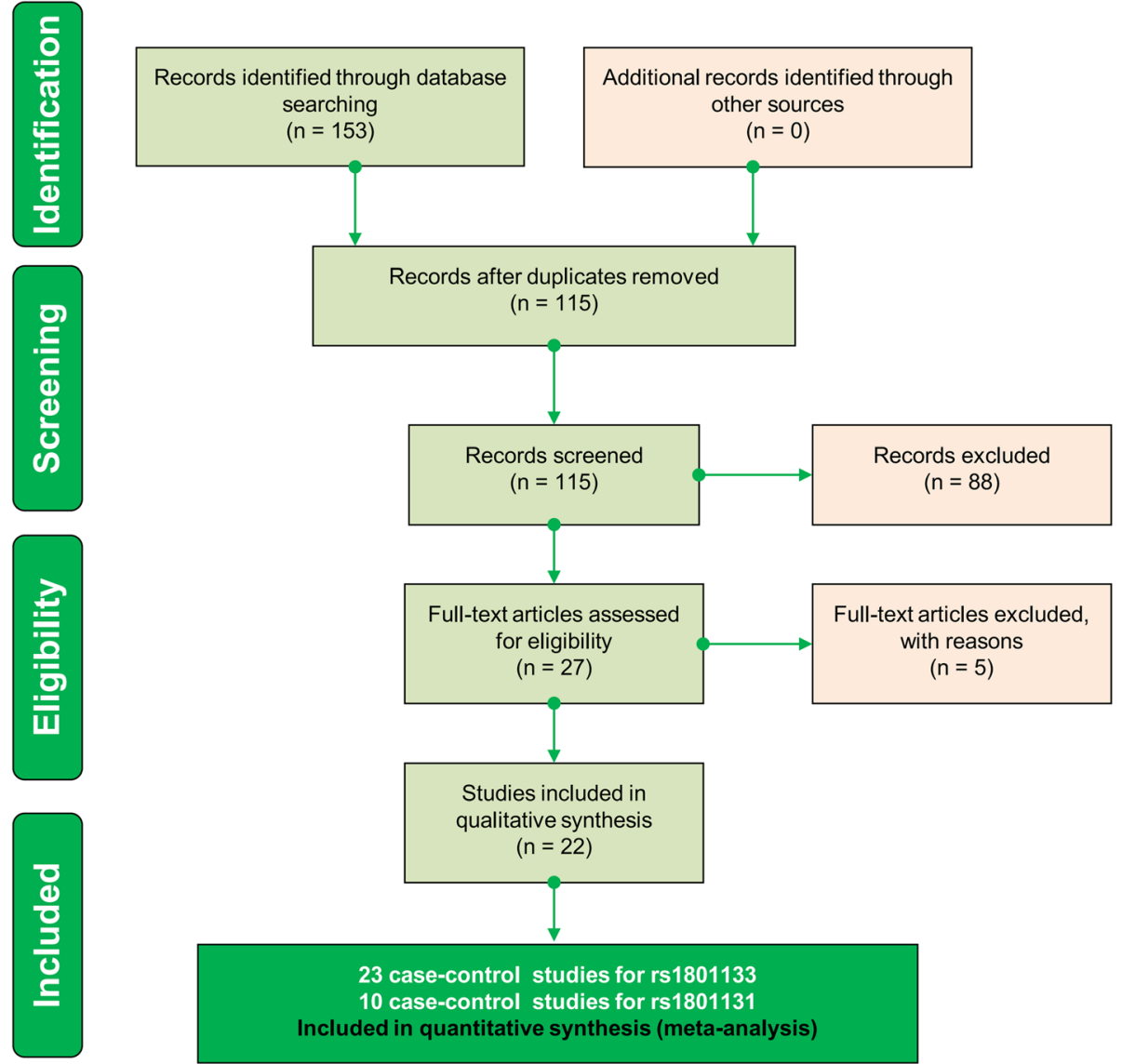

FIG. 1 Flowchart of database searching and record screening process 
Table 2 Pooled analysis for the MTHFR rs1801133 polymorphism

\begin{tabular}{|c|c|c|c|c|c|c|}
\hline \multirow[t]{2}{*}{ Comparison } & \multirow[t]{2}{*}{ Subgroup } & \multicolumn{2}{|c|}{ Sample size } & \multicolumn{3}{|c|}{ Test of association } \\
\hline & & Studies & Case/control & RRs (95\% Cls) & $z$ & P \\
\hline \multirow[t]{8}{*}{ allele T vs. allele C } & overall & 23 & $8321 / 17,731$ & $0.98(0.95,1.01)$ & 1.42 & 0.156 \\
\hline & UK & 6 & 1353/1517 & $0.99(0.92,1.07)$ & 0.14 & 0.886 \\
\hline & USA & 8 & $2863 / 4343$ & $1.00(0.95,1.05)$ & 0.14 & 0.890 \\
\hline & Japan & 3 & 1369/1933 & $0.97(0.91,1.03)$ & 1.03 & 0.301 \\
\hline & Caucasian & 18 & $6868 / 15,610$ & $0.99(0.96,1.02)$ & 0.86 & 0.391 \\
\hline & Asian & 5 & $1453 / 2121$ & $0.95(0.90,1.01)$ & 1.53 & 0.126 \\
\hline & hyperplastic polyps & 2 & $213 / 702$ & $0.99(0.84,1.16)$ & 0.13 & 0.897 \\
\hline & adenomatous polyps & 20 & 8086/16,994 & $0.98(0.95,1.01)$ & 1.43 & 0.153 \\
\hline \multirow[t]{8}{*}{ TT vs. CC } & overall & 22 & 8317/17,696 & $0.97(0.90,1.05)$ & 0.75 & 0.454 \\
\hline & UK & 6 & $1353 / 1517$ & $1.05(0.85,1.30)$ & 0.47 & 0.641 \\
\hline & USA & 7 & $2841 / 4308$ & $1.01(0.89,1.14)$ & 0.11 & 0.913 \\
\hline & Japan & 3 & 1369/1933 & $0.95(0.82,1.11)$ & 0.61 & 0.540 \\
\hline & Caucasian & 17 & $6846 / 15,575$ & $0.99(0.91,1.08)$ & 0.31 & 0.760 \\
\hline & Asian & 5 & $1453 / 2121$ & $0.92(0.80,1.07)$ & 1.06 & 0.291 \\
\hline & hyperplastic polyps & 2 & $213 / 702$ & $1.13(0.77,1.65)$ & 0.62 & 0.532 \\
\hline & adenomatous polyps & 20 & $8086 / 16,994$ & $0.97(0.77,1.65)$ & 0.88 & 0.377 \\
\hline \multirow[t]{8}{*}{ CT vs. CC } & overall & 23 & $8321 / 17,731$ & $0.97(0.94,1.00)$ & 1.77 & 0.077 \\
\hline & UK & 6 & $1353 / 1517$ & $0.96(0.89,1.04)$ & 0.93 & 0.351 \\
\hline & USA & 8 & $2863 / 4343$ & $0.99(0.94,1.04)$ & 0.44 & 0.663 \\
\hline & Japan & 3 & $1369 / 1933$ & $0.94(0.88,1.01)$ & 1.67 & 0.094 \\
\hline & Caucasian & 18 & $6868 / 15,610$ & $0.98(0.95,1.01)$ & 1.11 & 0.269 \\
\hline & Asian & 5 & $1453 / 2121$ & $0.94(0.87,1.00)$ & 1.92 & 0.055 \\
\hline & hyperplastic polyps & 2 & 213/702 & $0.88(0.73,1.07)$ & 1.27 & 0.205 \\
\hline & adenomatous polyps & 20 & $8086 / 16,994$ & $0.98(0.95,1.01)$ & 1.58 & 0.113 \\
\hline \multirow[t]{8}{*}{$C T+\pi$ vs. CC } & overall & 23 & $8321 / 17,731$ & $0.98(0.95,1.00)$ & 1.76 & 0.079 \\
\hline & UK & 6 & 1353/1517 & $0.98(0.91,1.06)$ & 0.66 & 0.511 \\
\hline & USA & 8 & $2863 / 4343$ & $0.99(0.95,1.04)$ & 0.33 & 0.743 \\
\hline & Japan & 3 & 1369/1933 & $0.96(0.91,1.01)$ & 1.53 & 0.125 \\
\hline & Caucasian & 18 & $6868 / 15,610$ & $0.98(0.96,1.01)$ & 1.08 & 0.280 \\
\hline & Asian & 5 & $1453 / 2121$ & $0.95(0.90,1.00)$ & 1.95 & 0.052 \\
\hline & hyperplastic polyps & 2 & 213/702 & $0.94(0.80,1.09)$ & 0.82 & 0.414 \\
\hline & adenomatous polyps & 20 & $8086 / 16,994$ & $0.98(0.95,1.00)$ & 1.65 & 0.098 \\
\hline \multirow[t]{8}{*}{ TT vs. CC + CT } & overall & 22 & $8317 / 17,696$ & $0.99(0.92,1.07)$ & 0.19 & 0.847 \\
\hline & UK & 6 & 1353/1517 & $1.09(0.87,1.36)$ & 078 & 0.436 \\
\hline & USA & 7 & $2841 / 4308$ & $1.02(0.89,1.16)$ & 0.23 & 0.822 \\
\hline & Japan & 3 & $1369 / 1933$ & $1.01(0.86,1.18)$ & 0.08 & 0.934 \\
\hline & Caucasian & 17 & $6846 / 15,575$ & $1.00(0.91,1.09)$ & 0.07 & 0.944 \\
\hline & Asian & 5 & $1453 / 2121$ & $0.98(0.83,1.15)$ & 0.28 & 0.780 \\
\hline & hyperplastic polyps & 2 & $213 / 702$ & $1.23(0.83,1.84)$ & 1.04 & 0.299 \\
\hline & adenomatous polyps & 20 & $8086 / 16,994$ & $0.98(0.91,1.84)$ & 0.39 & 0.696 \\
\hline \multirow[t]{8}{*}{ carrier T vs. carrier C } & overall & 23 & $8321 / 17,731$ & $0.99(0.96,1.01)$ & 0.99 & 0.322 \\
\hline & UK & 6 & $1353 / 1517$ & $0.99(0.92,1.07)$ & 0.21 & 0.831 \\
\hline & USA & 8 & $2863 / 4343$ & $1.00(0.95,1.05)$ & 0.15 & 0.883 \\
\hline & Japan & 3 & $1369 / 1933$ & $0.98(0.91,1.05)$ & 0.69 & 0.491 \\
\hline & Caucasian & 18 & $6868 / 15,610$ & $0.99(0.96,1.02)$ & 0.64 & 0.523 \\
\hline & Asian & 5 & $1453 / 2121$ & $0.97(0.90,1.03)$ & 0.99 & 0.322 \\
\hline & hyperplastic polyps & 2 & 213/702 & $0.98(0.82,1.16)$ & 0.26 & 0.793 \\
\hline & adenomatous polyps & 20 & $8086 / 16,994$ & $0.99(0.96,1.02)$ & 0.97 & 0.331 \\
\hline
\end{tabular}


study in which the TT genotype frequency of case and control groups for rs1801133 equaled zero was not included in the meta-analysis under the TT vs. CC (homozygote) and TT vs. CC + CT (recessive) models. The PRISMA-based analysis flowchart is shown in Fig. 1. None of the included studies exhibited poor quality (all NOS scores were larger than five).

\section{Pooled analysis for MTHFR rs 1801133}

First, we carried out a meta-analysis to investigate the genetic relationship between MTHFR rs1801133 and colorectal polyp susceptibility. A total of twenty-three case-control studies with 8321 cases and 17,731 controls were included. As shown in Table 2, compared with the control group, no increased risk of colorectal polyps was detected in the case group under the six genetic models, namely, allele $\mathrm{T}$ vs. allele $C$ ( $P$ value in test of association $=0.156)$; TT vs. CC $(P=0.454) ; C$ T vs. CC $(P=0.077) ; C \mathrm{CT}+\mathrm{TT}$ vs. CC $(P=0.079)$; TT vs. CC + CT $(P=0.847)$; carrier T vs. carrier $\mathrm{C}(P=0.322)$. We also conducted subgroup analyses by country, ethnicity (Caucasian/Asian) and disease type (hyperplastic polyps/adenomatous polyps). A similar nonsignificant genetic relationship was observed for all the models (all $P>0.05$, Table 2). For example, there was no significant difference between the colorectal polyp cases and negative controls in the $\mathrm{UK}$ subgroup under the $\mathrm{T}$ vs. $\mathrm{C}$ allele (Table $2, P=0.886)$; TT vs. CC $(P=0.641)$; CT vs. CC $(P=0.351) ; \mathrm{CT}+\mathrm{TT}$ vs. $\mathrm{CC}(P=0.511) ; \mathrm{TT}$ vs. $\mathrm{CC}+$ CT $(P=0.436)$; or carrier T vs. carrier C $(P=0.831)$. In the subgroup analysis of "adenomatous polyps", we also did not observe a statistically significant association under the allele $\mathrm{T}$ vs. allele $\mathrm{C}$ (Table 2, $P=$ $0.153)$; TT vs. CC $(P=0.377)$; $C$ T vs. $C C(P=0.113)$; $\mathrm{CT}+\mathrm{TT}$ vs. $\mathrm{CC} \quad(P=0.098) ; \mathrm{TT}$ vs. $\mathrm{CC}+\mathrm{CT} \quad(P=$ $0.696)$; and carrier $\mathrm{T}$ vs. carrier $\mathrm{C}(P=0.331)$. We show the forest plots of the subgroup analyses based on disease type under the allele $\mathrm{T}$ vs. allele $\mathrm{C}$ model in Fig. 2. These results revealed that MTHFR rs1801133 does not appear to be significantly linked to susceptibility to colorectal polyps.

\begin{tabular}{|c|c|c|}
\hline $\begin{array}{l}\text { Study } \\
\text { ID }\end{array}$ & $\mathrm{RR}(95 \% \mathrm{Cl})$ & $\begin{array}{l}\% \\
\text { Weight }\end{array}$ \\
\hline \multicolumn{3}{|l|}{ adenomatous polyps } \\
\hline Al-Ghnaniem (2007) & $0.74(0.43,1.27)$ & 0.39 \\
\hline Beckett (2015) & $0.94(0.69,1.29)$ & 0.85 \\
\hline Chen (1998) & $1.13(0.98,1.29)$ & 3.66 \\
\hline Chiang (2015) & $0.62(0.42,0.91)$ & 0.91 \\
\hline de Vogel (2011) & $0.96(0.91,1.02)$ & 25.66 \\
\hline Delgado (2001) & $1.13(0.86,1.50)$ & 0.68 \\
\hline Giovannucci (2003) & $0.98(0.87,1.10)$ & 5.42 \\
\hline Goode (2004) & $0.97(0.85,1.09)$ & 5.22 \\
\hline Hazra (2007) & $1.03(0.92,1.16)$ & 5.44 \\
\hline Hirose (2005) & $0.97(0.88,1.07)$ & 7.33 \\
\hline Kum (2006) & $1.39(0.57,3.40)$ & 0.08 \\
\hline Levine (2000) & $0.98(0.85,1.12)$ & 4.29 \\
\hline Lightfoot (2008) & $1.07(0.91,1.25)$ & 2.97 \\
\hline Marugame (2000) & $1.04(0.87,1.24)$ & 2.29 \\
\hline Mitrou (2006) & $0.97(0.88,1.07)$ & 8.69 \\
\hline Pufulete (2003) & $0.90(0.55,1.47)$ & 0.39 \\
\hline Ulrich (1999) & $0.94(0.83,1.06)$ & 5.64 \\
\hline van den (2005) & $1.00(0.91,1.11)$ & 7.27 \\
\hline Williams (2013) & $1.13(0.84,1.50)$ & 0.88 \\
\hline Yamaji (2009) & $0.95(0.87,1.03)$ & 8.82 \\
\hline Subtotal $(I-$ squared $=0.0 \%, p=0.569)$ & $0.98(0.95,1.01)$ & 96.89 \\
\hline \multicolumn{3}{|l|}{ hyperplastic polyps } \\
\hline Al-Ghnaniem (2007) & $0.98(0.53,1.82)$ & 0.23 \\
\hline Ulrich (2000) & $0.99(0.84,1.17)$ & 2.88 \\
\hline Subtotal $(I-$ squared $=0.0 \%, p=0.978)$ & $0.99(0.84,1.16)$ & 3.11 \\
\hline Overall $(\mathrm{I}$-squared $=0.0 \%, p=0.692$ ) & $0.98(0.95,1.01)$ & 100.00 \\
\hline
\end{tabular}

FIG. 2 Subgroup analysis by disease type of association between MTHFR rs1801133 polymorphism and colorectal polyp risk under the allele T vS. allele $\mathrm{C}$ model 


\section{Pooled analysis for MTHFR rs 1801131}

Next, ten studies containing 2951 cases and 3527 controls were included in the meta-analysis of MTHFR rs1801131. Pooled analysis in the overall population (Table 3) indicated a null association under all genetic models (all $P>0.05$ ). The results of the subgroup analysis for the UK, containing five studies of 1257 cases/ 1407 controls, suggested an increased risk in cases of colorectal polyps compared with controls under the genetic models of CC vs. AA $(P=0.032, \mathrm{RR}=1.27,95 \%$ $\mathrm{CIs}=1.02,1.57)$ and $\mathrm{CC}$ vs. $\mathrm{AA}+\mathrm{AC}(P=0.036, \mathrm{RR}=$ $1.27,95 \% \mathrm{CIs}=1.02,1.60)$. We showed the related forest plots in Figs. 3 and 4. Nevertheless, no difference between cases and controls was observed in other subgroup meta-analyses (all $P>0.05$, Table 3 ). For example, no increased or decreased risk of adenomatous polyps in cases was detected, compared with controls, under the allele $C$ vs. allele A (Table $3, P=0.138)$; $C$ vs. AA $(P=$ $0.114) ; \mathrm{AC}$ vs. AA $(P=0.576) ; \mathrm{AC}+\mathrm{CC}$ vs. AA $(P=$ $0.303)$; $\mathrm{CC}$ vs. $\mathrm{AA}+\mathrm{AC}(P=0.122)$; or carrier $\mathrm{T}$ vs. carrier
C $(P=0.376)$. Thus, the $\mathrm{C} / \mathrm{C}$ genotype of the MTHFR rs1801131 polymorphism may be related to an enhanced colorectal polyp risk in the UK population.

\section{Heterogeneity, publication bias and sensitivity analysis} In addition, we evaluated the between-study heterogeneity and did not detect remarkable heterogeneity in any of the above comparisons (Table 4 , all $\mathrm{I}^{2}<$ $50.0 \%, P$ value of heterogeneity $>0.1)$. Thus, a fixed-effects model was applied. We also conducted both Begg's test and Egger's test to assess the presence of publication bias. As shown in Table 4, the $P$ values of Begg's test and Egger's test were larger than 0.05 in all genetic models, indicating the absence of large publication bias. We showed Begg's funnel plot and the association between the MTHFR rs1801131 polymorphism and colorectal polyp risk under the CC vs. AA model in Fig. 5a. Additionally, similar pooled RRs were detected in our sensitivity analysis under other genetic models (Fig. $5 \mathrm{~b}$ for $\mathrm{CC}$ vs. AA model of

Table 3 Pooled analysis for the MTHFR rs 1801131 polymorphism

\begin{tabular}{|c|c|c|c|c|c|c|}
\hline \multirow[t]{2}{*}{ Comparison } & \multirow[t]{2}{*}{ Subgroup } & \multicolumn{2}{|c|}{ Sample size } & \multicolumn{3}{|c|}{ Test of association } \\
\hline & & Studies & case/control & RRs (95\% Cls) & z & $P$ \\
\hline \multirow[t]{4}{*}{ allele C vs. allele A } & overall & 10 & $2951 / 3527$ & $1.05(0.99,1.11)$ & 1.60 & 0.109 \\
\hline & UK & 5 & $1257 / 1407$ & $1.08(0.99,1.17)$ & 1.79 & 0.073 \\
\hline & Caucasian & 8 & $2225 / 2858$ & $1.04(0.98,1.10)$ & 1.22 & 0.222 \\
\hline & adenomatous polyps & 9 & $2934 / 3451$ & $1.04(0.99,1.10)$ & 1.48 & 0.138 \\
\hline \multirow[t]{4}{*}{ CC vs. AA } & overall & 10 & $2951 / 3527$ & $1.15(0.98,1.35)$ & 1.69 & 0.091 \\
\hline & UK & 5 & $1257 / 1407$ & $1.27(1.02,1.57)$ & 2.14 & 0.032 \\
\hline & Caucasian & 8 & $2225 / 2858$ & $1.14(0.96,1.35)$ & 1.50 & 0.133 \\
\hline & adenomatous polyps & 9 & $2934 / 3451$ & $1.14(0.97,1.34)$ & 1.58 & 0.114 \\
\hline \multirow[t]{4}{*}{$A C$ vs. AA } & overall & 10 & $2951 / 3527$ & $1.02(0.96,1.08)$ & 0.63 & 0.528 \\
\hline & UK & 5 & $1257 / 1407$ & $1.02(0.93,1.11)$ & 0.39 & 0.698 \\
\hline & Caucasian & 8 & $2225 / 2858$ & $1.01(0.95,1.07)$ & 0.25 & 0.805 \\
\hline & adenomatous polyps & 9 & $2934 / 3451$ & $1.02(0.96,1.08)$ & 0.56 & 0.576 \\
\hline \multirow[t]{4}{*}{$A C+C C$ vs. $A A$} & overall & 10 & $2951 / 3527$ & $1.03(0.98,1.08)$ & 1.13 & 0.258 \\
\hline & UK & 5 & $1257 / 1407$ & $1.04(0.97,1.12)$ & 1.08 & 0.279 \\
\hline & Caucasian & 8 & $2225 / 2858$ & $1.02(0.97,1.08)$ & 0.72 & 0.471 \\
\hline & adenomatous polyps & 9 & $2934 / 3451$ & $1.03(0.98,1.08)$ & 1.03 & 0.303 \\
\hline \multirow[t]{4}{*}{$C C$ vs. $A A+A C$} & overall & 10 & $2951 / 3527$ & $1.15(0.97,1.36)$ & 1.64 & 0.100 \\
\hline & UK & 5 & $1257 / 1407$ & $1.27(1.02,1.60)$ & 2.10 & 0.036 \\
\hline & Caucasian & 8 & $2225 / 2858$ & $1.14(0.96,1.36)$ & 1.49 & 0.135 \\
\hline & adenomatous polyps & 9 & $2934 / 3451$ & $1.14(0.97,1.35)$ & 1.55 & 0.122 \\
\hline \multirow[t]{4}{*}{ carrier C vs. carrier A } & overall & 10 & $2951 / 3527$ & $1.03(0.97,1.09)$ & 0.96 & 0.336 \\
\hline & UK & 5 & $1257 / 1407$ & $1.04(0.96,1.14)$ & 1.00 & 0.318 \\
\hline & Caucasian & 8 & $2225 / 2858$ & $1.02(0.96,1.09)$ & 0.68 & 0.499 \\
\hline & adenomatous polyps & 9 & $2934 / 3451$ & $1.03(0.97,1.09)$ & 0.88 & 0.376 \\
\hline
\end{tabular}

PB Population-based control, HB Hospital-based control, RRs Risk ratios, Cls Confidence intervals Bold entries are significant 


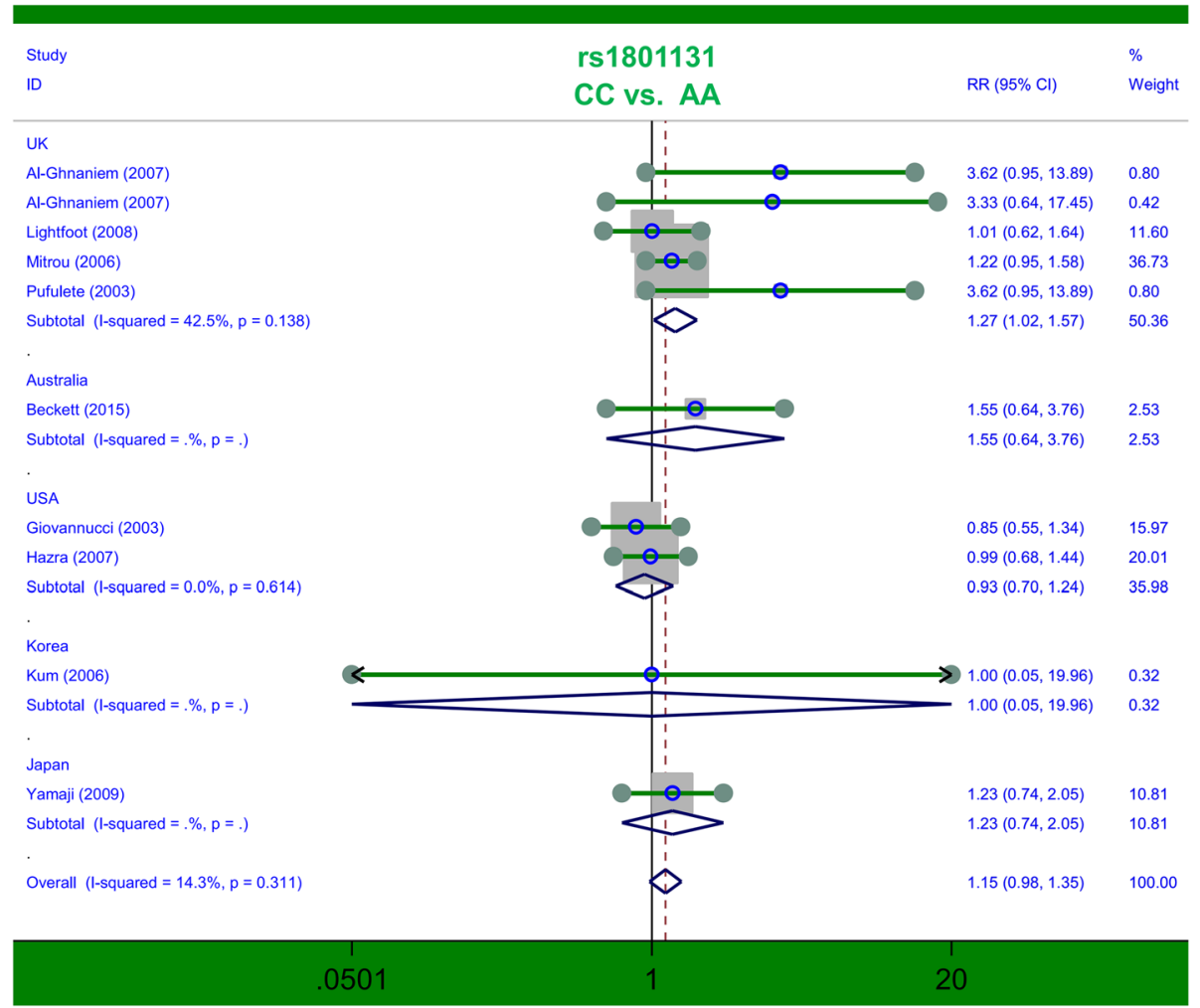

FIG. 3 Subgroup analysis by country of association between MTHFR rs1801131 polymorphism and colorectal polyp risk under the CC vs. AA model

MTHFR rs1801131; other data not shown), suggesting the reliability of pooling outcomes.

\section{Discussion}

Several meta-analyses have reported the role of MTHFR polymorphisms in the susceptibility to colorectal cancer (CRC) and adenoma. For example, in 2005, Kono, S. and colleague included a total of 16 case-control studies for a meta-analysis on the genetic relationship between MTHFR rs1801133 polymorphism and the risk of colorectal cancer and reported the potential role of the TT genotype in reduced CRC susceptibility [11]. In 2007, Huang, Y. et al. performed another meta-analysis to report that MTHFR rs1801133 and rs1801131 polymorphisms may confer reduced susceptibility to CRC patients [6]. In 2011, Zacho, J. et al. enrolled 75,000 cases and 93,000 controls for a meta-analysis of the association between the MTHFR rs1801133 polymorphism and overall cancer susceptibility and found that the TT genotype of MTHFR rs1801133 was associated with a decreased risk in CRC patients with lifelong hyperhomocysteinemia and hence hypomethylation [33]. Recently, data from another updated meta-analysis with 37,049 cases and 52,444 controls from 91 case-control studies suggested that the MTHFR rs1801133 polymorphism was related to a reduced risk of CRC, particularly in the Asian population [34]. These data supported the protective effect of MTHFR polymorphism, especially rs1801133, on CRC risk. However, inconsistent results regarding the role of the MTHFR polymorphism in the risk of colorectal adenoma were observed in the quantitative synthesis.

Meta-analysis of Huang, $Y$. et al. revealed that MTHFR rs1801133 and rs1801131 polymorphisms may have no increasing or decreasing effect on the risk of colorectal adenoma patients [6]. In addition, Edwards, T. L. and colleagues included 2551 colorectal adenoma cases and 3285 controls in the Caucasian population and performed genome-wide association studies (GWASs) to identify potential susceptibility factors, but MTHFR polymorphisms did not reach a genome-wide significant $P$ value [35]. However, Kono, $\mathrm{S}$. and colleagues reported that the TT genotype of the MTHFR rs1801133 polymorphism may be associated with high susceptibility to colorectal adenoma in patients with poor folate status [11]. In 2016, Montazeri, Z. and colleague conducted a systematic review and meta-analyses to assess the association between 37 polymorphisms within 26 genes and colorectal 


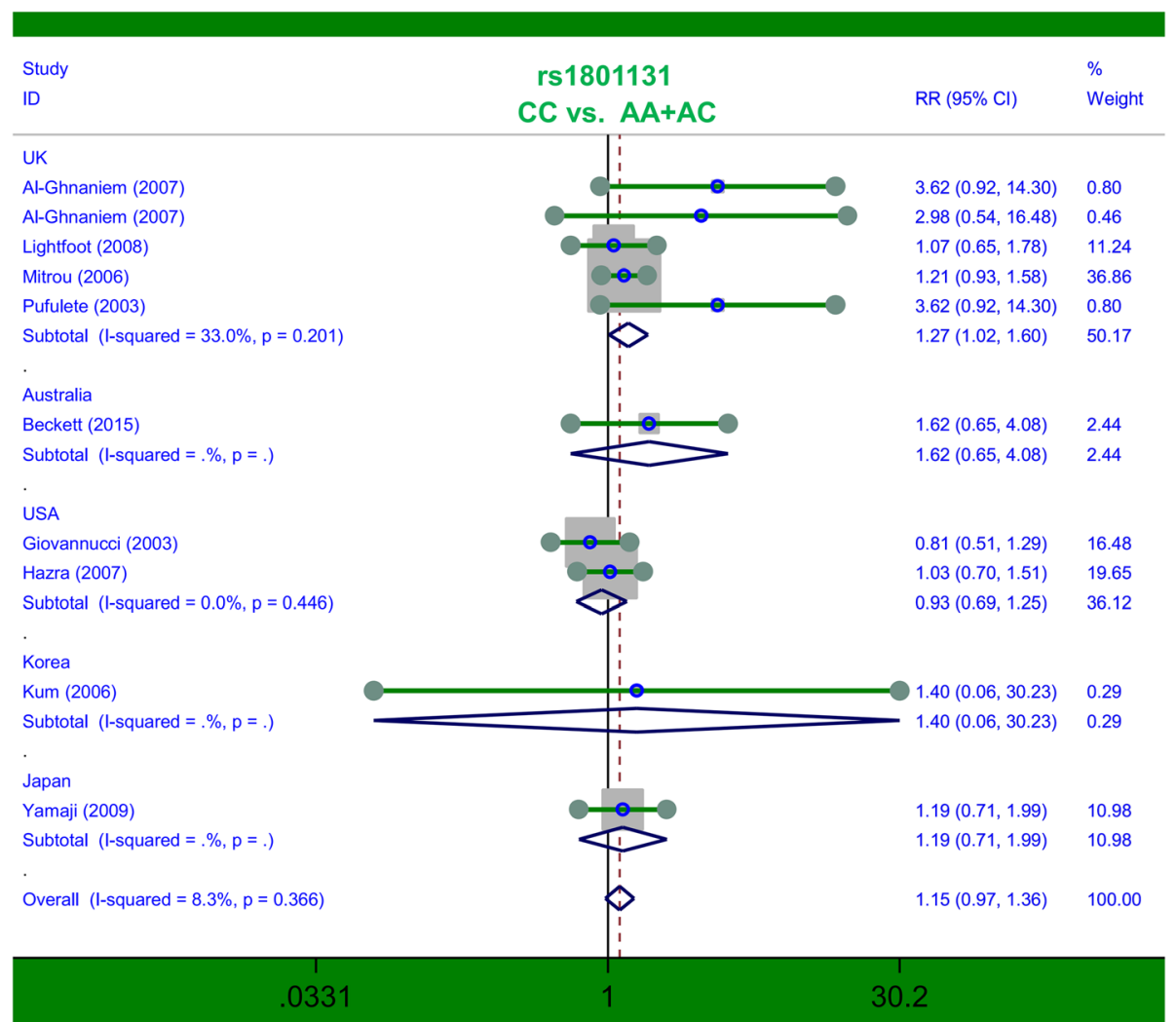

FIG. 4 Subgroup analysis by country of association between MTHFR rs 1801131 polymorphism and colorectal polyp risk under the CC vs. AA+AC model

adenoma risk and observed the potential genetic role of the MTHFR rs1801133 polymorphism, but with a relatively lower statistical power [12].

In this study, we intended to reassess the role of the MTHFR rs1801133 polymorphism in the susceptibility to colorectal adenomas in terms of colorectal polyps by means of a meta-analysis containing twenty-three case-control studies with 8339 cases and 17,731 controls. Our findings did not show any association between the MTHFR rs1801133 polymorphism and the risk of colorectal adenomatous polyps or hyperplastic polyps.

Moreover, we performed another meta-analysis of ten case-control studies with 2969 cases and 3527 controls and found that the $\mathrm{C} / \mathrm{C}$ genotype of the MTHFR rs1801131 polymorphism has a significant influence on an increased risk of colorectal polyps in the UK population. The A to $\mathrm{C}$ substitution in exon seven of MTHFR gene-induced abnormal enzymatic activity, homocysteine or folate level and DNA methylation/synthesis may be implicated in this process. It is noteworthy that, based on the requirement of meta-analysis for the enrolled case-control number, we evaluated only the subgroup analysis data with at least three case-control studies. Therefore, the subgroup analysis data for Australia, the USA, Korea, and Japan, with one or two case-control studies, exhibits very limited statistical power. We still cannot exclude the potential effect of the MTHFR rs1801131 polymorphism in colorectal polyp patients of other regions.

The case-control studies in our analysis were screened by fulfilling our strict selection criteria. All the studies exhibit high quality. In addition, we observed no heterogeneity in any of the Mantel-Haenszel statistics and excluded the large publication bias. Moreover, the stability of the statistical outcomes was detected by the sensitivity analysis. Nevertheless, we are also aware of several limitations. The main problem is the small sample size in the included case-control studies. For example, only one case-control study analyzed the correlation between the MTHFR rs1801131 polymorphism and hyperplastic polyp risk [14]. Second, only two SNPs were measured in our study. We did not study the genetic effects of other SNPs, combination with other genes, or the levels of folate, homocysteine, vitamin B12 and colorectal polyp risk. Third, hyperplastic and adenomatous polyps have complex and different etiologies. As a genetic effect of 
Table 4 The assessment of heterogeneity and publication bias

\begin{tabular}{|c|c|c|c|c|c|c|c|c|}
\hline \multirow[t]{2}{*}{ polymorphism } & \multirow[t]{2}{*}{ Comparison } & \multirow[t]{2}{*}{$1^{2}$} & \multirow{2}{*}{$\begin{array}{l}P \\
\text { value }\end{array}$} & \multirow[t]{2}{*}{ Model } & \multicolumn{2}{|c|}{ Begg's test } & \multicolumn{2}{|c|}{ Egger's test } \\
\hline & & & & & $z$ & $P$ & $t$ & $P$ \\
\hline \multirow[t]{6}{*}{ rs1801133 } & allele T vs. allele C & $0.0 \%$ & 0.736 & Fixed & 0.69 & 0.492 & 0.46 & 0.651 \\
\hline & TT vs. CC & $0.0 \%$ & 0.799 & Fixed & 0.90 & 0.367 & 0.75 & 0.463 \\
\hline & CT vs. CC & $0.0 \%$ & 0.705 & Fixed & 0.79 & 0.428 & -0.41 & 0.685 \\
\hline & $C T+\pi T$ vs. $C C$ & $0.0 \%$ & 0.725 & Fixed & 0.11 & 0.916 & -0.02 & 0.984 \\
\hline & TT vs. $C C+C T$ & $0.0 \%$ & 0.790 & Fixed & 0.73 & 0.463 & 0.70 & 0.492 \\
\hline & carrier $T$ vs. carrier $C$ & $0.0 \%$ & 0.999 & Fixed & 0.32 & 0.751 & 0.27 & 0.787 \\
\hline \multirow[t]{6}{*}{ rs1801131 } & allele C vs. allele A & $9.6 \%$ & 0.354 & Fixed & 1.16 & 0.245 & 1.41 & 0.195 \\
\hline & CC vs. AA & $14.3 \%$ & 0.311 & Fixed & 1.52 & 0.128 & 1.96 & 0.085 \\
\hline & $A C$ vs. AA & $0.0 \%$ & 0.800 & Fixed & 0.45 & 0.655 & -0.25 & 0.807 \\
\hline & $A C+C C$ vs. $A A$ & $0.0 \%$ & 0.623 & Fixed & 1.34 & 0.180 & 0.64 & 0.541 \\
\hline & $C C$ vs. $A A+A C$ & $8.3 \%$ & 0.366 & Fixed & 1.52 & 0.128 & 2.17 & 0.061 \\
\hline & carrier $C$ vs. carrier A & $0.0 \%$ & 0.918 & Fixed & 0.98 & 0.325 & 1.04 & 0.327 \\
\hline
\end{tabular}

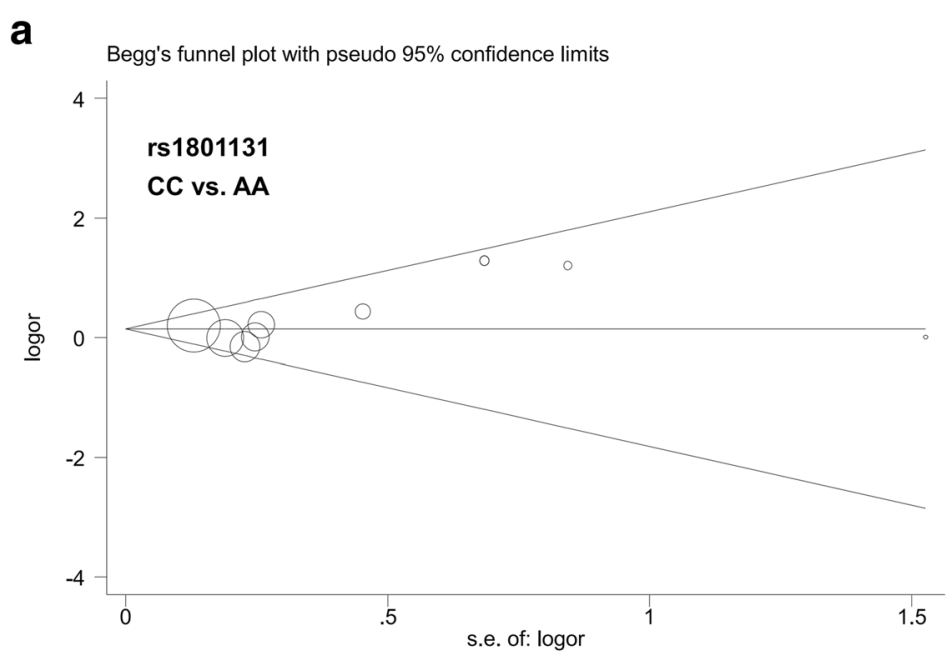

b

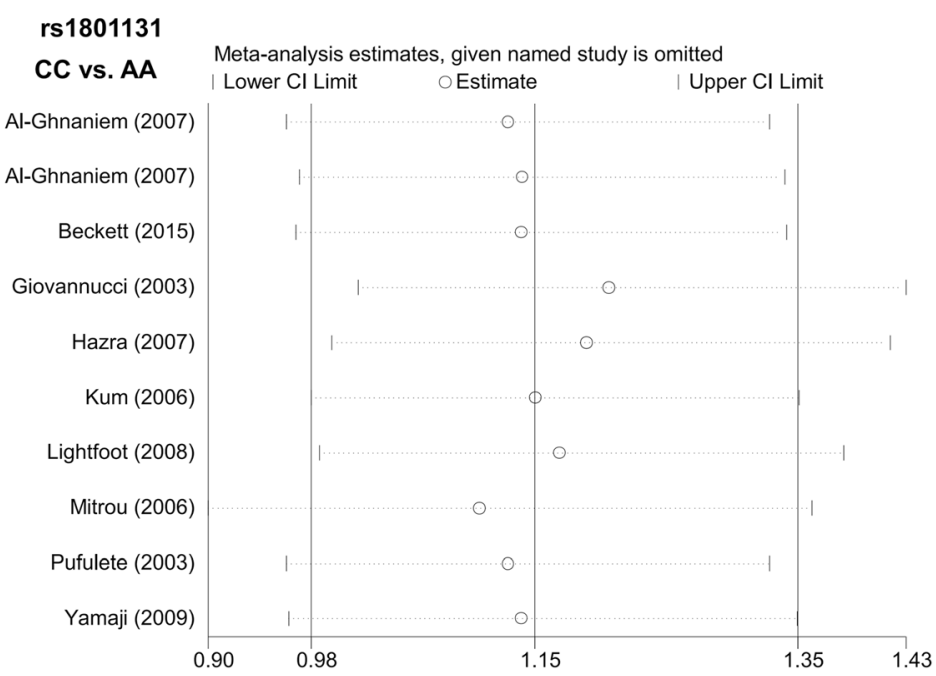

FIG. 5 Begg's funnel plot and sensitivity analysis for MTHFR rs 1801131 polymorphism and colorectal polyp risk under the CC vs. AA model. a Begg's funnel plot; $\mathbf{b}$ Sensitivity analysis 
MTHFR rs1801133 and rs1801131 polymorphisms has been suggested in the susceptibility to colorectal cancer $[6,11,33,34]$, additional confounding factors such as smoking, drinking, age, sex, and patient features should be adjusted for further investigation of the MTHFR variants in the malignant conversion from colorectal polyp.

\section{Conclusion}

Taken together, our findings conclude that MTHFR rs1801131, rather than rs1801133, is more likely to be associated with an increased susceptibility to colorectal polyps in the UK population. Additionally, the $\mathrm{C} / \mathrm{C}$ genotype of MTHFR rs1801131 may confer an increased susceptibility to patients with colorectal polyps in the UK region. However, this conclusion merits further confirmation with a larger sample size.

\section{Additional file}

Additional file 1: Table S1. The search terms used with the PubMed WOS and EMBASE databases. (DOCX $30 \mathrm{~kb}$ )

\section{Abbreviations}

Cl: Confidence interval; CRC: Colorectal cancer; FAP: Familial adenomatous polyposis; GWAS: Genome-wide association studies; HWE: Hardy-Weinberg Equilibrium; MTHFR: Methylenetetrahydrofolate reductase; NOS: NewcastleOttawa Scale; RRs: Risk ratios

\section{Acknowledgments}

We appreciate American Journal Experts (https://www.aje.com/) for help with English usage during the preparation of this manuscript.

\section{Funding}

Not applicable.

\section{Availability of data and materials}

All data generated or analyzed during the present study are included in this published article.

\section{Authors' contributions}

MS and JZ conceived and designed the study. MS and JZ performed the database searching and study screening. MS, JZ, LZ and SS extracted, analyzed, and interpreted the data. MS and JZ drafted the manuscript. All authors have read and approved the final manuscript.

\section{Ethics approval and consent to participate}

Not applicable.

\section{Consent for publication}

Not applicable.

\section{Competing interests}

The authors declare that they have no competing interests.

\section{Publisher's Note}

Springer Nature remains neutral with regard to jurisdictional claims in published maps and institutional affiliations.

\section{Author details}

'Department of Gastroenterology, Tianjin Union Medical Center, No.190, Jieyuan Road, Hongqiao District, Tianjin 300121, People's Republic of China. ${ }^{2}$ Department of Radiology, Tianjin Union Medical Center, Tianjin 300121 ,
People's Republic of China. ${ }^{3}$ Department of Pathology, Tianjin Union Medical Center, Tianjin 300121, People's Republic of China.

Received: 3 October 2018 Accepted: 9 May 2019

Published online: 30 May 2019

\section{References}

1. Angarita FA, Feinberg $A E$, Feinberg $S M$, Riddell RH, McCart JA. Management of complex polyps of the colon and rectum. Int J Color Dis. 2018;33(2):115-29.

2. Geramizadeh B, Robertson S. Serrated polyps of Colon and Rectum: a Clinicopathologic review. J Gastrointest Cancer. 2017;48(4):291-8.

3. Anderson JC, Lisovsky M, Greene MA, Hagen C, Srivastava A. Factors associated with classification of hyperplastic polyps as sessile serrated adenomas/polyps on morphologic review. J Clin Gastroenterol. 2018; 52(6):524-9.

4. ML DEM, Tonelli F, Quaresmini D, Lovero D, Della-Morte D, Silvestris F, Guadagni F, Palmirotta R. Desmoid tumors in familial adenomatous polyposis. Anticancer Res. 2017;37(7):3357-66.

5. Hiraoka M, Kagawa Y. Genetic polymorphisms and folate status. Congenit Anom (Kyoto). 2017:57(5):142-9.

6. Huang Y, Han S, Li Y, Mao Y, Xie Y. Different roles of MTHFR C677T and A1298C polymorphisms in colorectal adenoma and colorectal cancer: a meta-analysis. J Hum Genet. 2007:52(1):73-85.

7. Sun MY, Zhang L, Shi SL, Lin JN. Associations between methylenetetrahydrofolate reductase (MTHFR) polymorphisms and nonalcoholic fatty liver disease (NAFLD) risk: a meta-analysis. PLoS One. 2016; 11(4):e0154337.

8. Yi KH, Baik HW, Jang EJ, Park SJ, Park JS, Park YM, Moon WC. Epigenetic risk factors of colorectal adenoma in Korean. J Cancer Prev. 2006;11(3):171-5.

9. van den Donk M, Buijsse B, van den Berg SW, Ocke MC, Harryvan JL, Nagengast FM, Kok FJ, Kampman E. Dietary intake of folate and riboflavin, MTHFR C677T genotype, and colorectal adenoma risk: a Dutch case-control study. Cancer Epidemiol Biomark Prev. 2005;14(6):1562-6.

10. Marugame T, Tsuji E, Inoue H, Shinomiya S, Kiyohara C, Onuma K, Hamada $H$, Koga $H$, Handa K, Hayabuchi $H$, et al. Methylenetetrahydrofolate reductase polymorphism and risk of colorectal adenomas. Cancer Lett. 2000; 151(2):181-6.

11. Kono S, Chen K. Genetic polymorphisms of methylenetetrahydrofolate reductase and colorectal cancer and adenoma. Cancer Sci. 2005;96(9): 535-42.

12. Montazeri Z, Theodoratou E, Nyiraneza C, Timofeeva M, Chen W, Svinti V, Sivakumaran S, Gresham G, Cubitt L, Carvajal-Carmona L, et al. Systematic meta-analyses and field synopsis of genetic association studies in colorectal adenomas. Int J Epidemiol. 2016;45(1):186-205.

13. Moher D, Liberati A, Tetzlaff J, Altman DG. Preferred reporting items for systematic reviews and meta-analyses: the PRISMA statement. PLoS Med. 2009:6(7):e1000097.

14. Al-Ghnaniem R, Peters J, Foresti R, Heaton N, Pufulete M. Methylation of estrogen receptor alpha and mutL homolog 1 in normal colonic mucosa: association with folate and vitamin B-12 status in subjects with and without colorectal neoplasia. Am J Clin Nutr. 2007;86(4):1064-72.

15. Ashktorab H, Begum R, Akhgar A, Smoot DT, Elbedawi M, Daremipouran $M$, Zhao A, Momen B, Giardiello FM. Folate status and risk of colorectal polyps in African Americans. Dig Dis Sci. 2007:52(6):1462-70.

16. Beckett EL, Martin C, Choi JH, King K, Niblett S, Boyd L, Duesing K, Yates Z, Veysey M, Lucock M. Folate status, folate-related genes and serum miR-21 expression: implications for miR-21 as a biomarker. BBA Clin. 2015;4:45-51.

17. Chen J, Giovannucci E, Hankinson SE, Ma J, Willett WC, Spiegelman D, Kelsey KT, Hunter DJ. A prospective study of methylenetetrahydrofolate reductase and methionine synthase gene polymorphisms, and risk of colorectal adenoma. Carcinogenesis. 1998;19(12):2129-32.

18. Chiang FF, Huang SC, Wang HM, Chen FP, Huang YC. High serum folate might have a potential dual effect on risk of colorectal cancer. Clin Nutr. 2015:34(5):986-90.

19. de Vogel S, Schneede J, Ueland PM, Vollset SE, Meyer K, Fredriksen A, Midttun O, Bjorge T, Kampman E, Bretthauer M, et al. Biomarkers related to one-carbon metabolism as potential risk factors for distal colorectal adenomas. Cancer Epidemiol Biomark Prev. 2011;20(8):1726-35.

20. Delgado-Enciso I, Martinez-Garza SG, Rojas-Martinez A, Ortiz-Lopez R, Bosques-Padilla F, Calderon-Garciduenas AL, Zarate-Gomez M, Barrera- 
Saldana HA. 677T mutation of the MTHFR gene in adenomas and colorectal cancer in a population sample from the northeastern Mexico. Preliminary results. Rev Gastroenterol Mex. 2001;66(1):32-7.

21. Giovannucci E, Chen J, Smith-Warner SA, Rimm EB, Fuchs CS, Palomeque C, Willett WC, Hunter DJ. Methylenetetrahydrofolate reductase, alcohol dehydrogenase, diet, and risk of colorectal adenomas. Cancer Epidemiol Biomark Prev. 2003;12(10):970-9.

22. Goode EL, Potter JD, Bigler J, Ulrich CM. Methionine synthase D919G polymorphism, folate metabolism, and colorectal adenoma risk. Cancer Epidemiol Biomark Prev. 2004;13(1):157-62.

23. Hazra A, Wu K, Kraft P, Fuchs CS, Giovannucci EL, Hunter DJ. Twenty-four non-synonymous polymorphisms in the one-carbon metabolic pathway and risk of colorectal adenoma in the Nurses' health study. Carcinogenesis. 2007;28(7):1510-9.

24. Hirose M, Kono S, Tabata S, Ogawa S, Yamaguchi K, Mineshita M, Hagiwara T, Yin G, Lee KY, Tsuji A, et al. Genetic polymorphisms of methylenetetrahydrofolate reductase and aldehyde dehydrogenase 2 , alcohol use and risk of colorectal adenomas: self-defense forces health study. Cancer Sci. 2005;96(8):513-8.

25. Levine AJ, Siegmund KD, Ervin CM, Diep A, Lee ER, Frankl HD, Haile RW. The methylenetetrahydrofolate reductase $677 \mathrm{C} \rightarrow>$ T polymorphism and distal colorectal adenoma risk. Cancer Epidemiol Biomark Prev. 2000;9(7):657-63.

26. Lightfoot TJ, Barrett JH, Bishop T, Northwood EL, Smith G, Wilkie MJ, Steele RJ, Carey FA, Key $T$, Wolf $R$, et al. Methylene tetrahydrofolate reductase genotype modifies the chemopreventive effect of folate in colorectal adenoma, but not colorectal cancer. Cancer Epidemiol Biomark Prev. 2008:17(9):2421-30.

27. Mitrou PN, Watson MA, Loktionov AS, Cardwell C, Gunter MJ, Atkin WS, Macklin CP, Cecil T, Bishop TD, Primrose J, et al. MTHFR (C677T and A1298C) polymorphisms and risk of sporadic distal colorectal adenoma in the UK flexible sigmoidoscopy screening trial (United Kingdom). Cancer Causes Control. 2006;17(6):793-801.

28. Pufulete M, Al-Ghnaniem R, Leather AJM, Appleby P, Gout S, Terry C, Emery PW, Sanders TAB. Folate status, genomic DNA hypomethylation, and risk of colorectal adenoma and cancer: a case control study. Gastroenterology. 2003;124(5):1240-8.

29. Ulrich CM, Kampman E, Bigler J, Schwartz SM, Chen C, Bostick R, Fosdick L, Beresford SA, Yasui Y, Potter JD. Colorectal adenomas and the C677T MTHFR polymorphism: evidence for gene-environment interaction? Cancer Epidemiol Biomark Prev. 1999;8(8):659-68.

30. Ulrich CM, Kampman E, Bigler J, Schwartz SM, Chen C, Bostick R, Fosdick L, Beresford SA, Yasui Y, Potter JD. Lack of association between the C677T MTHFR polymorphism and colorectal hyperplastic polyps. Cancer Epidemiol Biomark Prev. 2000;9(4):427-33.

31. Williams EA, Welfare M, Spiers A, Hill MH, Bal W, Gibney ER, Duckworth Y, Powers HJ, Mathers JC. Systemic folate status, rectal mucosal folate concentration and dietary intake in patients at differential risk of bowel cancer (the FAB2 study). Eur J Nutr. 2013;52(7):1801-10.

32. Yamaji T, Iwasaki M, Sasazuki S, Sakamoto H, Yoshida T, Tsugane S Methionine synthase A2756G polymorphism interacts with alcohol and folate intake to influence the risk of colorectal adenoma. Cancer Epidemiol Biomark Prev. 2009;18(1):267-74.

33. Zacho J, Yazdanyar S, Bojesen SE, Tybjaerg-Hansen A, Nordestgaard BG. Hyperhomocysteinemia, methylenetetrahydrofolate reductase c.677C $>$ T polymorphism and risk of cancer: cross-sectional and prospective studies and meta-analyses of 75,000 cases and 93,000 controls. Int J Cancer. 2011; 128(3):644-52

34. Xu L, Qin Z, Wang F, Si S, Li L, Lin P, Han X, Cai X, Yang H, Gu Y. Methylenetetrahydrofolate reductase C677T polymorphism and colorectal cancer susceptibility: a meta-analysis. Biosci Rep. 2017;37(6):1-15. https://doi org/10.1042/BSR20170917.

35. Edwards TL, Shrubsole MJ, Cai Q, Li G, Dai Q, Rex DK, Ulbright TM, Fu Z, Delahanty RH, Murff HJ, et al. Genome-wide association study identifies possible genetic risk factors for colorectal adenomas. Cancer Epidemiol Biomark Prev. 2013;22(7):1219-26.

Ready to submit your research? Choose BMC and benefit from:

- fast, convenient online submission

- thorough peer review by experienced researchers in your field

- rapid publication on acceptance

- support for research data, including large and complex data types

- gold Open Access which fosters wider collaboration and increased citations

- maximum visibility for your research: over $100 \mathrm{M}$ website views per year

At BMC, research is always in progress.

Learn more biomedcentral.com/submissions 